\title{
衣藻细胞色素 $b_{559} \alpha$ 亚基 $\operatorname{Ser}^{24}$ 残基定点突变影响PS II 光 合放氧活性
}

\author{
马晶晶 ${ }^{(2)}$ 李良璧 ${ }^{(1}$ 荆玉祥 ${ }^{(1)}$ 匡廷云 ${ }^{(* *}$ \\ (1) 中国科学院植物研究所光合作用与环境分子生理学重点实验室, 北京 100093; (2) 中国科学院研究生院, 北京 100049.*联系人, \\ E-mail: kuangty@ibcas.ac.cn)
}

摘要 为研究细胞色素 $b_{559}\left(\mathrm{Cyt} b_{559}\right)$ 在光合系统中的功能, 采用大引物定点突变技术, 将衣藻叶绿体 Cyt $b_{559} \alpha$ 亚基 $\left(p s b E\right.$ 基因编码)中与组氨酸 $\left(\mathrm{His}^{23}\right)$ 相邻的氨基酸残基丝氨酸 $\left(\mathrm{Ser}^{24}\right)$ 定点突变为苯丙氨酸 (Phe), 获得突变体S24F. 对突变体的生理生化分析表明, S24F既能进行光合自养也能进行光合异养, 但 是在自养和异养条件下, 其生长速率比对照慢; S24F的PS II 放氧活性约为野生衣藻细胞的 $71 \%$, S24F的 光系统 II (PS II) 光化学效率 $F_{\mathrm{v}} / F_{\mathrm{m}}$ 比野生型对照低约 0.23 . 此外, 相比野生型对照, 突变体 $S 24 F$ 对强光 更加敏感; SDS-PAGE和Western杂交的结果表明, 对Cyt $b_{559} \alpha$ 亚基中 $\mathrm{Ser}^{24}$ 的定点突变影响了Cyt $b_{559} \alpha$ 亚基及其他膜蛋白，如 LHC II 和PsbO等的表达. 上述结果说明，虽然 $\mathrm{Ser}^{24}$ 残基并不参与血红素配位，但 其对维持PS II 的活性具有重要作用.

关键词 Cyt $b_{559}$ 定点突变 PSII 叶绿体转化 衣藻

光系统 II (PS II) 是存在于高等植物、藻类和蓝细 菌类囊体膜上的一种色素蛋白复合体, 是进行水氧 化、质体醌还原的重要场所 ${ }^{[1]}$, 也是光合电子传递链 的重要组成部分 ${ }^{[2,3]}$. PS II 核心是反应中心复合蛋白, 它由D1, D2, Psb I 以及一个跨膜 $b$ 型色素蛋白, 即细 胞色素 $b_{559}\left(\mathrm{Cyt} b_{559}\right)$ 组成 ${ }^{[4]}$.

Cyt $b_{559}$ 是一个血红素蛋白, 存在于所有放氧光 合生物的PS II 反应中心(PS II -RC)复合物中, 由 $\alpha$ 亚 基 $(9.3 \mathrm{kD})$ 和 $\beta$ 亚基 $(4.4 \mathrm{kD})$ 两个多肽以及一个血红素 组成. 两个亚基的化学计量为 1 ：1, 各含有一个跨膜 $\alpha$ 螺旋. $\alpha$ 和 $\beta$ 亚基氨基酸残基分别为 83 和 49 个(其中 各含一个组氨酸残基), 分别由叶绿体基因组中的 $p s b E$ 和 $p s b F$ 编码 $[5]$. 综合亲水性分析和遗传学及光谱 学分析的结果, 推测血红素分别与 $\alpha$ 和 $\beta$ 亚基的His残 基交联, 所处的平面与膜平面垂直. Cyt $b_{559}$ 在PS II 中 的位置靠近 D1/D2 $2^{[6]}$. Zouni 等人 ${ }^{[7]}$ 对嗜热蓝细菌 Synechococcus elongates PS II 晶体结构的研究, 进一 步明确了Cyt $b_{559}$ 卟啉铁在PS II 中, 处在距离 $\mathrm{ChlzD}_{2}$ 蛋白 $2.7 \mathrm{~nm}$, 距离膜质侧约 $0.8 \mathrm{~nm}$ 的空间位置.

在过去的 20 多年中, 对Cyt $b_{559}$ 在PS II 中的功能 研究取得了很大进展. 对高等植物中分离纯化的PS II - RC蛋白复合物的研究表明, 要使 PS II 维持光
合活性, Cyt $b_{559}$ 的存在是必不可少的. 研究者推测, Cyt $b_{559}$ 可能具有以下几个功能: Cyt $b_{559}$ 具有对PS II 的光保护作用, 通过Cyt $b_{559}$ 的环式电子流耗散PS II 中的多余激发能而参与其受体侧及供体侧光抑制的 防御 ${ }^{[8,9]}$; Cyt $b_{559}$ 是组成稳定的PS II 的必需组分 ${ }^{[10,11]}$; 此外, Cyt $b_{559}$ 还参与了水的氧化还原 ${ }^{[12]}$; 近年还有报 道称Cyt $b_{559}$ 能够提供电子给质体醌 ${ }^{[13]}$. 然而由于 $\mathrm{Cyt}$ $b_{559}$ 的复杂特性以及相关Cyt $b_{559}$ 突变体往往缺乏高 活性PS II, 其许多功能至今仍不清楚 ${ }^{[11]}$.

以往对Cyt $b_{559}$ 的研究重点主要在Cyt $b_{559}$ 中与血 红素连接的His残基上 ${ }^{[10,11,14,15]}$, 其他氨基酸残基对 Cyt $b_{559}$ 功能影响的研究很少. 到目前为止, 仅有 Bondarava等人 ${ }^{[13]}$ 用篮选到的烟草突变株, 分析了 Cyt $b_{559} \beta$ 链紧中靠近组氨酸的苯丙氨酸(Phe)被丝氨 酸(Ser)取代后光合特性及PSII活性的变化. 本研究 采用定点突变技术, 以衣藻为实验材料, 将 Cyt $b_{559} \alpha$ 亚基中 $\mathrm{His}^{23}$ 下游相邻的氨基酸 $\mathrm{Ser}^{24}$ 用Phe取代, 获得 衣藻突变体S24F. 研究结果显示, 靠近血红素配位环 境的氨基酸 $\mathrm{Ser}^{24}$, 对维持 Cyt $b_{559}$ 的正常功能, 组装 有活性的PS II 有重要作用.

\section{1 材料与方法}

( i ) 衣藻及其培养. 衣藻(Chlamydomonas re- 
inhadtii)野生型CC-125 $\mathrm{mt}^{+}$, 美国杜克大学衣藻基因 中心Harris教授(CGC, Department of Botany, Duke University, Durham, NC) 馈赠. 野生型衣藻在温度 $20 \sim 25^{\circ} \mathrm{C}$, 光照 $50 \sim 100 \mu \mathrm{mol} \cdot \mathrm{m}^{-2} \cdot \mathrm{s}^{-1}$ 的条件下于TAP (Tris-acetate-phosphate)或HSM (high salt minimal)液 体培养基中生长, 每天光照时间为 $8 \mathrm{~h}^{[16]}$.

(ii) 利用大引物突变法进行定点突变. (1) 质 粒. 质粒p78 和p423 由Harris教授馈赠. 质粒p78 中含 有衣藻叶绿体基因组的Pst I -4 片段(15.1 kb, 包含 $P s b E$ 基因及其上下游序列), 质粒 $\mathrm{p} 423$ 含有 $a a d A$ 表达 框, 可以提供壮观霉素(spectinomycin)抗性筛选标记. 质粒pE3 克隆有 $p s b E$ 基因和 $a a d A$ 表达框，在质粒 $\mathrm{p} 78$ 和p423 的基础上构建而成 ${ }^{[17]}$. (2) 定点突变. 定点突 变的方法如Landt等人 ${ }^{[18]}$ 所述, 具体步骤见图 1. 所用 引物为 P1 (5'-TTACGATGTGCCAGAAA-3') 和 P2 (5'-TTTAATTTGAACTTGAGCTACCGCTTC-

TTTACGAC-3'), 突变引物 MS24F序列为 5'-AAGCAGGTACCGTAATAAAGTGAATAACCC-3'(下画线 示新构建的限制性内切酶 Kpn I 的酶切位点, 斜体为 Phe的密码子). PCR反应在 MJ PTC-200 (Bio-Rad Laboratories, Inc, Waltham, MA)上进 行, 高保真Pfu DNA聚合酶购自上海 生工生物工程技术有限责任公司 (上海 生工). 约 $1.6 \mathrm{~kb}$ 的PCR终产物用 $S a c$ I 和Acc I 双酶切, 回收 $0.8 \mathrm{~kb}$ 片段并与 Sac I /Acc I 双酶切质粒pE3 后回收的 大片段相连接, 构建叶绿体同源重组 质粒 (命名为 $\mathrm{pS} 24 \mathrm{~F}$ ). $\mathrm{pS} 24 \mathrm{~F}$ 送上海生 工测序确认突变位点.

(iii) 衣藻叶绿体遗传转化和转基 因衣藻的篮选. 采用基因枪转化法对 衣藻叶绿体进行遗传转化, 具体操作 参照Boynton和 Gillham ${ }^{[19]}$ 的方法进行. 使用BioRad Biolistic PDS-1000/He基 因枪将带有突变位点的质粒pS24F转 化到衣藻叶绿体中. 由于转化质粒pE3 的阳性对照与野生型衣藻对照在生理 特性上基本没有区别 ${ }^{[11]}$, 因此在本研 究中直接以野生型衣藻作为对照. 转 基因衣藻在选择培养基(TAP+壮观雷 素(100 $\mu \mathrm{g} / \mathrm{ml})$ )上篮选 4 代后进行叶绿
体同质化鉴定.

(iv) 转基因衣藻的同质化鉴定. 按照Lee等人 [20]所描述的方法分别提取野生型衣藻和转基因衣藻 的叶绿体基因组 DNA. 以此为模板, 用 P3 (5'-ATAGATGGTTTGAAAAGG-3') 和 P4 (5'-TTGTTTCAATGGGGCATTTA-3')引物进行PCR扩增. $1 \%$ 琼脂糖电泳鉴定扩增产物. PCR-Southern杂交按照地 高辛标记试剂盒 I (Roche Diagnostics Gmbh, Germany) 的使用指南进行.

( v ) 衣藻生长速率及吸收值的测定. 衣藻生长 速率的测定参照沈萍等人 ${ }^{[21]}$ 的方法. 用紫外/可见分 光光度计 (model MPS-8500, Shimadzu, Kyoto, Japan) 在 $750 \mathrm{~nm}$ 处测定光吸收值.

( vi ) 衣藻放氧速率的测定. 具体方法参照 Leong ${ }^{[22]}$ 的方法进行. 用Clark-type氧电极(Hansatech, King's Lynn, UK) 在 $25^{\circ} \mathrm{C}$, 饱和光强 $(1000 \mu \mathrm{mol}$. $\left.\mathrm{m}^{-2} \cdot \mathrm{s}^{-1}\right)$ 下测量衣藻细胞的光合放氧率.

(vii) 衣藻苂光动力学参数的测定. 参照Gent等 人 ${ }^{[23]}$ 的方法, 略有改动. 衣藻细胞在TAP的液体培养

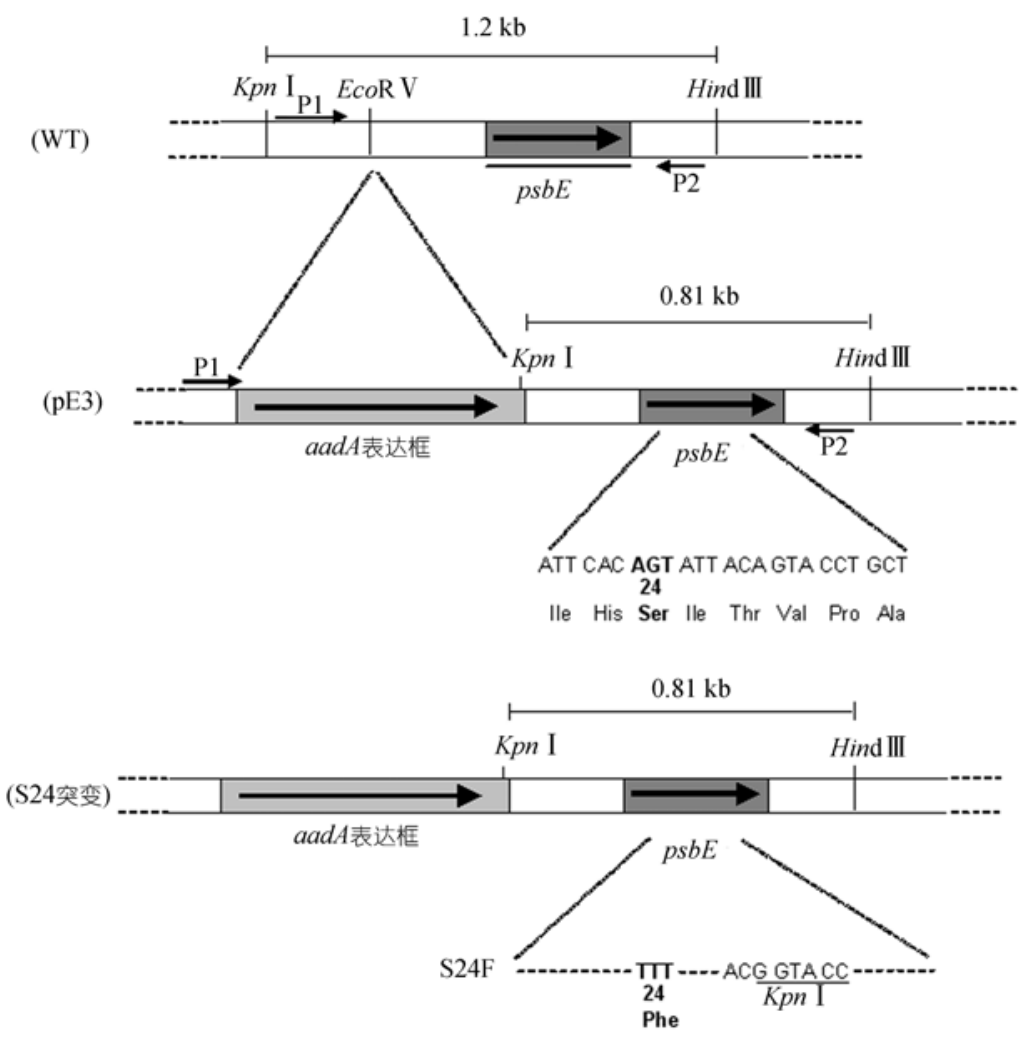

图 1 转化载体 $\mathrm{pS} 24 \mathrm{~F}$ 的构建 $a a d A$ 表达框和 $p s b E$ 的方向用箭头表示. 相关的定点突变的位置用黑体标出 
基中培养至 $A_{750}$ 为 $0.6 \sim 0.8$. 取出部分细胞, 暗适应 5 $\min$ 后, 室温下用脉冲调制式苂光仪(PAM-101, Walz, Germany)非常弱的测量光(光强为 $30 \mu \mathrm{mol} \cdot \mathrm{m}^{-2} \cdot \mathrm{s}^{-1}$ 左右) 测定叶绿素的最小苂光 $\left(F_{0}\right)$; 然后用饱和脉冲光 $(1000$ $\mu \mathrm{mol} \cdot \mathrm{m}^{-2} \cdot \mathrm{s}^{-1}$ )照射 $0.8 \mathrm{~s}$, 测定最大苂光 $\left(F_{\mathrm{m}}\right)$, 计算PS II 光 化学效率 $F_{\mathrm{v}} / F_{\mathrm{m}}\left[\left(F_{\mathrm{m}}-F_{0}\right) / F_{0}\right]$ 值. 每个样品重复 5 10 次.

(viii) 衣藻细胞对强光的敏感性测定. 将 $A_{750}$ 值 为 $0.6 \sim 0.8$ 的衣藻细胞分别放置在光强为 200 和 1000 $\mu \mathrm{mol} \cdot \mathrm{m}^{-2} \cdot \mathrm{s}^{-1}$ 的光源下, 每 $10 \mathrm{~min}$ 取 $3 \mathrm{~mL}$ 样品在饱和 光强 $\left(1000 \mu \mathrm{mol} \cdot \mathrm{m}^{-2} \cdot \mathrm{s}^{-1}\right)$ 下测细胞的放氧速率, 直到 持续光照 $30 \mathrm{~min}$ 为止.

(ix) 类囊体膜蛋白多肽组分 SDS-PAGE 电泳、 Western杂交和吸收光谱的测定. 衣藻类囊体膜蛋白多 肽组分的提取依照Ruffle等人 ${ }^{[24]}$ 的方法进行. 提取的类 囊体膜蛋白多肽组分进行SDS-PAGE电泳，一半胶用于 考马斯亮蓝R250 染色, 一半胶用于Western杂交分析. 蛋白质的SDS-PAGE电泳、Western杂交按照Sambrook 等人 ${ }^{[25]}$ 的方法进行, 所用抗体分别是Cyt $b_{559} \alpha$ 链单克 隆抗体(Barber教授馈赠, Department of Biochemistry, Imperial College of Science, Technology, and Medicine, London, United Kingdom)、LHC II 及 PsbO单克隆抗体 (中国科学院植物研究所张立新研究员馈赠). 每个 Western杂交条带的相对量用 Labworks 软件 (Ultra-Violet Products Ltd, America)进行测定(设 3 次重 复). 常温下, 采用UVKON-943 双光束分光光度计在避 光条件下进行类囊体膜蛋白多肽组分吸收光谱测定. 所用扫描速度 $100 \mathrm{~nm} / \mathrm{min}$, 分辨率 $0.5 \mathrm{~nm}$. 样品浓度调 至 $10 \mu \mathrm{g} \mathrm{Chl} / \mathrm{mL}$.

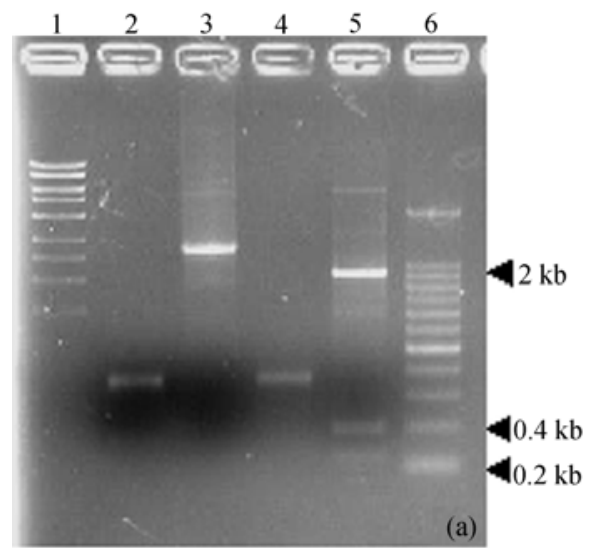

\section{2 结果与分析}

\section{1 叶绿体同源重组质粒 pS24F 的构建}

质粒 $\mathrm{pS} 24 \mathrm{~F}$ 的构建过程如下: 将 $1.6 \mathrm{~kb}$ 带有突变 位点的PCR产物用限制性内切酶Sac I, Acc I 双酶切, 将其中 $800 \mathrm{bp}$ 大小的酶切片段回收后克隆至质粒pE3 的S $a c$ I / A c I 位点, 得到带有突变位点的质粒pS24F. 质粒 $\mathrm{pS} 24 \mathrm{~F}$ 的 $p s b E$ 基因为 Cyt $b_{559} \alpha$ 多肽链在衣藻中 的编码基因, a adA 表达框为壮观霉素抗性筛选标记. 将质粒送上海生工测序, 测序结果表明在预定位点 发生了突变.

\section{2 转基因衣藻的篮选和同质化鉴定}

基因枪法转化后的衣藻(命名为 S24F)在含有壮 观霉素的选择培养基上篮选 4 代后, 分别提取野生型 衣藻和 S24F 的叶绿体基因组 DNA, 以 P3 和 P4 为引 物进行 PCR 扩增, 结果如图 2(a)所示. 引物 P3 和 P4 分别位于 $p s b E$ 基因表达框的上、下游, 以野生型衣 藻的叶绿体 DNA 为模板的 PCR 扩增产物为 $0.75 \mathrm{~kb}$ 大小的片段(图 2(a), 泳道 2); 以衣藻突变株 S24F 的 叶绿体基因组 DNA 为模板 PCR 扩增产物的大小为 $2.6 \mathrm{~kb}$ 左右(图 2(a), 泳道 3), 说明外源基因 $\operatorname{aadA}$ 表 达框 (大小为 $1.9 \mathrm{~kb}$ ) 已经整合到 $\mathrm{S} 24 \mathrm{~F}$ 的叶绿体基因 组中. 图 2(a)中泳道 3 的结果显示, 除了约 $2.6 \mathrm{~kb}$ 的 片段外, 扩增产物中没有 $0.75 \mathrm{~kb}$ 大小的片段, 说明 $\mathrm{S} 24 \mathrm{~F}$ 的叶绿体基因组是纯合的, 基本不含有未转化 的叶绿体基因组. Kpn I 酶切上述的 PCR 产物, 结果 显示只有在转基因藻的酶切产物中有 0.2 和 $0.4 \mathrm{~kb}$ 大 小的片段, 与预期的突变位点相符(图 2(a)泳道 4 和 5). 为了更加明确 PCR 扩增片段的特异性, 将图 2(a)

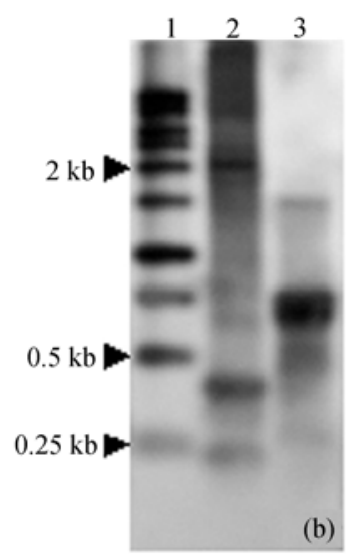

图 2 引物 P3 和 P4 对衣藻野生型和 S24F 叶绿体 DNA 的 PCR 和 PCR-Southern 杂交分析

(a) PCR 产物的电泳图谱: 1, DNA Ladder; 2 和 3, 野生型衣藻和 S24F PCR 产物; 4 和 5, Kpn I 分别酶切野生型衣藻和 S24F PCR 产物; 6, 200 bp DNA Ladder. (b) PCR-Southern 杂交分析:1, 1/kb DNA ladder; 2 和 3,S24F 和野生型衣藻 PCR 扩增产物 Kpn / 酶切后与 psbE 探针杂交的结果 
泳道 4 和 5 中的产物转到尼龙膜上, 与标记的 $p s b E$ 基因探针杂交. PCR-Southern 杂交的结果(图 2(b), 泳 道 2 和 3)与电泳结果完全符合. 上述结果表明含突变 位点的 $p s b E$ 基因已整合到突变体的叶绿体基因组中, 且突变体叶绿体的同质化程度很高, 基本不含未转 化的叶绿体.

\section{3 突变株 S24F 的生长和光合作用特性}

衣藻突变株S24F既能进行光合自养, 又能光合 异养, 但是生长速度相比野生型衣藻缓慢 (表 1). 突 变株到达生长平台期的时间在自养和异养条件下, 分别比野生型衣藻慢 4 和 $8 \mathrm{~d}$. S24F的PS II 光化学效 率 $F_{\mathrm{v}} F_{\mathrm{m}}$ 比野生型低约 0.23 , 其PS II 放氧活性约为野 生型的 $71 \%$.

\section{4 衣藻突变株 S24F 对强光的敏感性}

在中强度光 $\left(200 \mu \mathrm{mol} \cdot \mathrm{m}^{-2} \cdot \mathrm{s}^{-1}\right)$ 照射细胞时, 突变
株 S24F 的放氧活性迅速下降, 到 $30 \mathrm{~min}$ 时, 其放氧 活性仅为未处理时放氧活性的 $68 \%$ (表 2); 当用高强 度光 $\left(1000 \mu \mathrm{mol} \cdot \mathrm{m}^{-2} \cdot \mathrm{s}^{-1}\right)$ 照射细胞 $10 \mathrm{~min}$ 后, 突变株 $\mathrm{S} 24 \mathrm{~F}$ 放氧活性降为 0 , 而野生型衣藻在强光照射 30 min 后还保留 35\%的放氧活性. 这说明相比野生型衣 藻, 突变株 S24F 对强光表现更加敏感.

\section{$2.5 \mathrm{~S} 24 \mathrm{~F}$ 类囊体膜蛋白多肽组分分析}

S24F突变株与野生型对照的类囊体膜蛋白多肽 组分的吸收图谱相似, 有 3 个吸收峰, 分别位于 440 , 460 和 $678 \mathrm{~nm}$ 处(图 3(b)和(c)). S24F类囊体膜蛋白多 肽组分的SDS-PAGE结果(图 3(a))及Western杂交结果 (图 4)表明, 突变株S24F的类囊体膜蛋白各个多肽组 分都有表达, 但是 $\mathrm{Cyt} b_{559} \alpha$ 亚基、PsbO和LHC II 蛋白 的表达量相比对照都有下降, 其中PsbO和Cyt $b_{559} \alpha$ 亚基的表达量仅为野生型的 $80 \%$ 左右.

表 1 衣藻野生型和突变株 S24F 生长和光合特性

\begin{tabular}{|c|c|c|c|c|c|c|}
\hline \multirow{2}{*}{ 株系 } & \multicolumn{2}{|c|}{ 到达平台期的天数/d } & \multirow{2}{*}{$F_{0}$} & \multirow{2}{*}{$F_{\mathrm{m}}$} & \multirow{2}{*}{$F_{\mathrm{v}} / F_{\mathrm{m}}$} & \multirow{2}{*}{ 放氧率/ $/ \mu \mathrm{molO}_{2} \cdot \mathrm{mg}^{-1} \mathrm{Chl} \cdot \mathrm{h}^{-1}$} \\
\hline & HSM & TAP & & & & \\
\hline CC-125 & 4 & 8 & $0.257 \pm 0.048$ & $0.908 \pm 0.270$ & $0.706 \pm 0.045$ & $150 \pm 8.3$ \\
\hline S24F & 8 & 16 & $0.264 \pm 0.068$ & $0.514 \pm 0.125$ & $0.475 \pm 0.071$ & $106 \pm 10.2$ \\
\hline
\end{tabular}

表 2 突变株 S24F 对中强度光和高强度光的敏感性测定

\begin{tabular}{ccccc}
\hline 光照时间 $/ \mathrm{min}$ & \multicolumn{2}{c}{ 中强度光 $\left(200 \mu \mathrm{mol} \cdot \mathrm{m}^{-2} \cdot \mathrm{s}^{-1}\right)$} & \multicolumn{2}{c}{ 高强度光 $\left(1000 \mu \mathrm{mol} \cdot \mathrm{m}^{-2} \cdot \mathrm{s}^{-1}\right)$} \\
\cline { 2 - 4 } \cline { 5 - 6 } & $\mathrm{CC}-125$ & $\mathrm{~S} 24 \mathrm{~F} 24 \mathrm{~F}$ & $\mathrm{CC}-125$ & $106 \pm 10.2$ \\
10 & $150 \pm 8.3$ & $106 \pm 10.2$ & $150 \pm 8.3$ & 0 \\
20 & $147 \pm 7.7$ & $77 \pm 8.9$ & $137 \pm 4.26$ & 0 \\
30 & $143 \pm 12.3$ & $68 \pm 11.2$ & $123 \pm 7.56$ & 0 \\
\hline
\end{tabular}

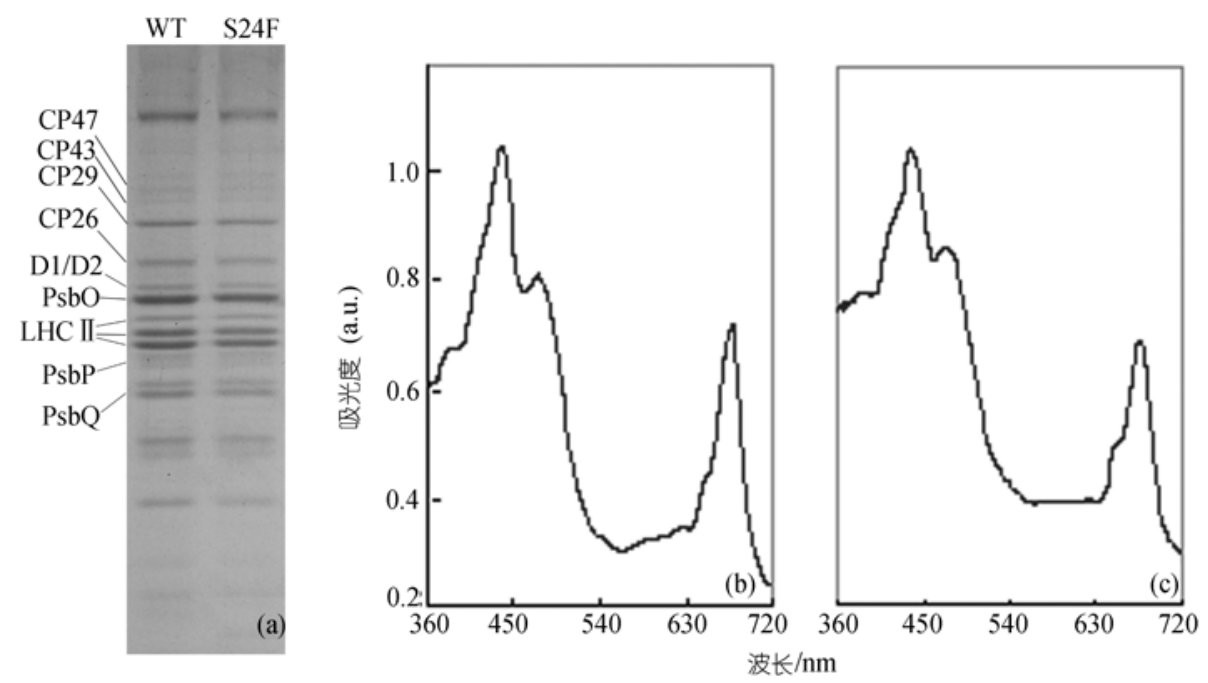

图 3 衣藻野生型和突变体 S24F 类囊体膜蛋白多肽组分 SDS-PAGE 分析和吸收光谱 (a) 衣藻野生型和 S24F 类囊体膜蛋白多肽组分的SDS-PAGE 分析!(b)和(c)3衣藻野生型和-S24F 类囊体膜蛋白多肽组分的吸收光谱图 


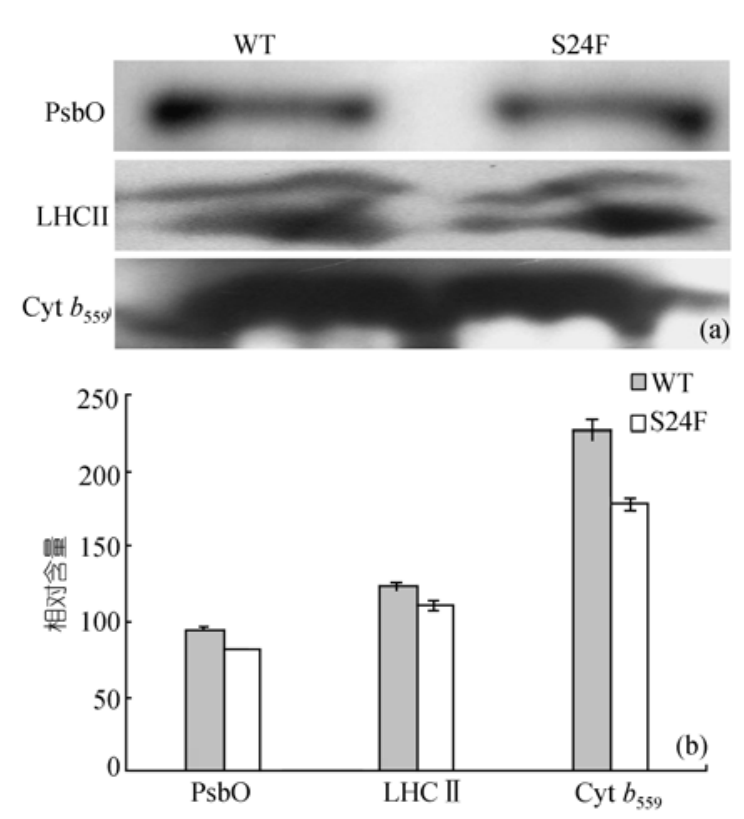

图 4 衣藻野生型和S24F的PsbO, LHC II 及Cyt $b_{559} \alpha$ 亚基 Western杂交的结果(a)及Western杂交条带相对量分析(b) 每个样品的上样量相当于 $3 \mu \mathrm{g} \mathrm{Chl}$

\section{3 讨论}

衣藻是一种单细胞绿色藻类, 其生活周期短, 既 能进行自养生长又能进行异养生长, 易于高效地进 行叶绿体基因组转化和核基因组转化的特性, 使它 成为研究真核生物生长发育分子机理的模式材料, 特别是在一些不能用酵母作为材料进行研究的领域, 如光合作用领域和叶绿体生物学研究领域等 ${ }^{[16]}$. 此 外, 在高等植物叶绿体和蓝藻中, 编码Cyt $b_{559} \alpha$ 和 $\beta$ 链的基因 $p s b E$ 和 $p s b F$ 与基因 $p s b L$ 和 $p s b J$ 组成一个操 纵子. 但在衣藻中, 这 4 个基因位于染色体的不同位 置, 并且编码方向相反 ${ }^{[26]}$, 因此对衣澡 $p s b E$ 基因的突 变不会影响另外 3 个基因, 这对构建其相关突变体提 供了良好的条件. 为此, 本研究采用衣藻作为实验材 料构建 Cyt $b_{559}$ 突变体.

Cyt $b_{559}$ 是存在于所有放氧光合生物PS II 中的一 个血红素蛋白, 类囊体膜中没有Cyt $b_{559}$ 就不能组成 稳定的PS II. 为了研究Cyt $b_{559}$ 的结构和功能, 人们 构建了许多突变体. 例如, 将蓝藻中 $p s b$ 和 $p s b F$ 分别 用 $\mathrm{Km}^{\mathrm{r}}$ 基因取代 ${ }^{[14,27]}$; 在衣藻 $p s b E$ 中插入 $\operatorname{aad} A$ 基因 [10]; 用亮氨基酸(Leu)分别或同时取代蓝藻Cyt $b_{559} \alpha$ 和 $\beta$ 亚基的 $\mathrm{His}^{22[15]}$ 以及用甲硫氨酸(Met)、酪氨酸(Tyr) 和谷氨酸取代衣藻Cyt $b_{559} \alpha$ 亚基的 $H i s^{23}$ 等 $[11]$. 然而
这些突变体往往严重影响组成PS II 蛋白的表达及PS II 活性, 甚至PS II 活性完全丧失. 由于缺乏有 高活性PS II 的Cyt $b_{559}$ 突变体, 使得 Cyt $b_{559}$ 许多功 能仍不清楚 [11].

此外, 上述 Cyt $b_{559}$ 突变体的定点突变研究也主 要侧重于与血红素相连的His残基, Cyt $b_{559}$ 中其他氨 基酸残基对 Cyt $b_{559}$ 功能的影响报道很少, 仅 Bondarava等曾报道在烟草中Cyt $b_{559} \beta$ 亚基紧靠His 的 $\mathrm{Phe}^{26}$ 被Ser取代后, PS II 活性约为对照的 $40 \%$ 1ㅣㄴ. 为此, 本研究通过大引物法定点突变技术, 用Phe取 代Cyt $b_{559} \alpha$ 亚基中紧靠 $\mathrm{His}^{23}$ 的 $\mathrm{Ser}^{24}$ 残基, 构建了 Cyt $b_{559}$ 突变体 $\mathrm{S} 24 \mathrm{~F}$. 突变体的生长和光合特性分析表明 (表 1), 该突变体既能光合自养, 也能光合异养, 但其 生长速度仅为野生型衣藻的一半左右; 此外, PS II 放氧 活性和光化学效率 $F_{\mathrm{v} /} F_{\mathrm{m}}$ 都比野生型细胞低, 说明对 $\mathrm{Ser}^{24}$ 的突变影响了突变体细胞的光合作用. S24F对强 光的敏感性测定结果表明(表 2), 相比对照, 突变体 对强光表现更加敏感. S24F的生长和光合作用特性及 对强光的敏感性与突变 $\mathrm{His}^{23}$ 后得到结果类似 ${ }^{[11]}$, 但 是其PS II 活性与烟草Phe突变体的PS II 活性比其他 Cyt $b_{559}$ 突变体的PS II 活性更高, 说明对 $\mathrm{Ser}^{24}$ 突变的 影响比对 $\mathrm{His}^{23}$ 突变弱, 这可能跟 $\mathrm{His}^{23}$ 直接与卟啉环 相连相关.

Cyt $b_{559}$ 的缺失突变以及对 $\alpha$ 或 $\beta$ 链中的His的突 变都会严重影响组成PS II 的多个蛋白组分, 如D1, $\mathrm{D} 2, \mathrm{CP} 47, \mathrm{PsbO}, \mathrm{Cyt} b_{559} \alpha$ 和 $\beta$ 亚基等的表达, PS II 不 能组装或者PS II 活性很低, 如Pakrasi等人 用 Leu分别或同时取代蓝藻 Cyt $b_{559}$ 中 $\alpha$ 和 $\beta$ 链中的 $\mathrm{His}^{22}$, 发现突变体中的 $\mathrm{D}_{1}, \mathrm{D}_{2}, \mathrm{CP} 43$ 和 CP47 降解, Cyt $b_{559} \alpha$ 亚基比野生型的小 $1.5 \mathrm{kD}$ (单点突变时); 当衣 藻中的Cyt $b_{559} \alpha$ 链中的 $\mathrm{His}^{23}$ 被氨基酸Met和Tyr取代 后, $\mathrm{D}_{1}, \mathrm{D}_{2}$ 蛋白和Cyt $b_{559} \alpha$ 及 $\beta$ 链的表达量分别仅为 野生型的 $10 \% \sim 25 \%$. 此外, Cyt $b_{559} \beta$ 链Phe ${ }^{26}$ 的突变 也减少了 CP47 和Cyt $b_{559} \alpha$ 和 $\beta$ 链的表达量, 并导致 PS II 在类囊体膜上的组装数量下降 ${ }^{[13]}$. SDS- PAGE 和Western杂交的结果表明(图 3 和 4), 对 $\mathrm{Ser}^{24}$ 的突变 也有类似的现象, 如LHC II 表达量降低, PsbO和 Cyt $b_{559} \alpha$ 亚基的表达量仅为野生型的 $80 \%$ 左右等. 但结 果也表明, 对 $\mathrm{Ser}^{24}$ 和 $\beta$ 链中 $\mathrm{Phe}^{26}$ 的突变影响PS II 蛋白 组分的程度小于对His突变造成的影响.

总之, 所有结果表明, 不与血红素直接相连的 $\mathrm{Ser}^{24}, 6$ 是 $C y \mathrm{t} \boldsymbol{b}_{55}$ 的行使功能所不可缺少的, 对于维 
持PS II 的活性也非常重要. 此外, 在后续工作中, 对 有较强PS II 活性的S24F的光氧化、光还原过程中的 电子传递反应、动力学特征做深入的研究, 也有助于 进一步了解 Cyt $b_{559}$ 在PS II 中的结构和功能.

致谢 感谢Imperial College of Science, Technology, and Medicine的Barber教授提供Cyt $b_{559} \alpha$ 亚基和中国科学院植 物研究所张立新研究员提供LHC II 及 PsbO单克隆抗体.

\section{参考文献}

1 Hankamer B, Barber J, Boekema E J. Structure and membrane organization of photosystem II in green plants. Annu Rev Plant Physiol Plant Mol Biol, 1997, 48: 641—671[DOI]

2 Aro E M, Virgin I, Andersson B. Photoinhibition of Photosystem II : Inactivation, protein damage and turnover. Biochim Biophys Acta, 1993, 1143: 113-134[DOI]

3 Barber J, Andersson B. Too much of a good thing: Light can be bad for photosynthesis. Trends Biochem Sci, 1992, 17: 61-66[DOI]

4 Satoh K. The Photosynthetic Reaction Centers. Orlando, FL: Academic Press, 1993. 289-318

5 Herrmann R G, Alt J, Schiller B, et al. Nucleotide sequence of the gene for apocytochrome b-559 on the spinach plastid chromosome: Implications for the structure of the membrane protein. FEBS Lett, 1984, 176: 239-244[DOI]

6 Nanba O, Satoh K. Isolation of a photosystem II reaction center consisting of D-1 and D-2 polypeptides and Cytochrome b-559. Proc Natl Acad Sci USA, 1987, 84: 109-112[DOI]

7 Zouni A, Witt H T, Kern J, et al. Crystal structure of photosystem II from Synchococcus elongatus at $3.8 \AA$ resolution. Nature, 2001, 409: 739-743[DOI]

8 Heber U, Kirk M R, Boardman N K. Photoreactions of cytochrome $b-559$ and cyclic electron flow in Photosystem II of intact chloroplasts. Biochim Biophys Acta, 1979, 546: 292-306

9 Thompson L K, Brudvig G W. Cytochrome $b$-559 may function to protect Photosystem II from photoinhibition. Biochemistry, 1988, 27: 6653-6658[DOI]

10 Morais F, Barber J, Nixon P J. The chloroplast-encoded $\alpha$ subunit of cytochrome b-559 is required for assembly of the photosystem two complex in both the light and the dark in Chlamydomonas reinhardtii. J Biol Chem, 1998, 273: 29315-29320[DOI]

11 Morais F, Barber J, Nixon P J. Photosynthetic water oxidation in cytochrome $b_{559}$ mutants containing a disrupted heme-binding pocket. J Biol Chem, 2001, $276: 31986$-31993[DOI]

12 Fiege R, Shuvalov V A. Correlated behavior of the EPR signal of cytochrome b-559 heme $\mathrm{Fe}(\mathrm{III})$ ligated by $\mathrm{OH}^{-}$and the multiline signal of the Mn cluster in PS- II membrane fragments. FEBS Lett, 1996, 387: 33-35[DOI]
13 Bondarava N, Pascalis L D, Al-Babili S, et al. Evidence that cytochrome b559 mediates the oxidation of reduced.plastoquinone in the dark. J Biol Chem, 2003, 278: 13554-13560[DOI]

14 Pakarasi H B, Diner B A, Williams J G K, et al. Deletion mutagenesis of the cytchrome b-559 protein inactivates the reaction center of photosystem II. Plant Cell, 1989, 1: 591-597[DOI]

15 Pakrasi H B, Ciechi P D, Whitmarsh J. Site directed mutagenesis of the heme axial ligands of cytochrome b-559 affects the stability of the Photosystem II complex. EMBO J, 1991, 10: 1619-1627

16 Harris E H. The Chlamydomonas Sourcebook. San Diego: Academic, 1989. 780

17 Ma J J. Site-directed mutagenesis of residue $\operatorname{Arg} 18$ and Ser24 in Cyt b-559 $\alpha$ subunit and effects on PS II activity. Doctor Dissertation. Beijing: The Institute of Botany, CAS, 2006. 46-49

18 Landt $\mathrm{O}$, Grunert H P, Hahn U. A general method for rapid site-directed mutagenesis using the polymerase chain reaction. Gene, 1990, 96: 125-128[DOI]

19 Boynton J E, Gillham N W. Chloroplast transformation in Chlamydomonas. Methods Enzymol, 1993, 217, 510-536

20 Lee H, Bingham S E, Webber A N. Specific Mutagenesis of reaction center proteins by chloroplast transformation of Chlamudomonas reinhadtii. Methods Enzymol, 1998, 297: 310-337.

21 沈萍, 范秀容, 李广武, 主编. 微生物学实验(第 3 版). 北京: 高 等教育出版社, 1999

22 Leong A. Adaption of the thylakoid membranes of pea chloroplasts to light intensities. II . Regulation of electron transport capacities, electron carriers, coupling factor (CF1) activity and rates of photosynthesis. Photosynth Res, 1984, 5: 117-128[DOI]

23 Genty B, Briantais J M, Baker N R. The relationship between the quantum yield of photosynthetic electron transport and quenching of chlorophyll fluorescence. Biophys Acta, 1989, 990: 87—92

24 Ruffle S V, Wang J, Johnston H G. Photosystem II peripheral accessory chlorophyll mutants in Chlamydomonas reinhardtii biochemical characterization and sensitivity to photo-inhibition. Plant Physiol, 2001, 127: 633-644[DOI]

25 Sambrook J, Fritsch E F, Maniatis J. Molecular Cloning: A Laboratory Manual, 2nd ed. New York: Cold Spring Harbor Laboratory Press, 1989. 672-733

26 Tsafir S M, Itzhak O, Joseph $\mathrm{H}$, et al. An unusual organization of the gene encoding cytchrome $b_{559}$ in Chlamydomonas reinhardtii: $\mathrm{psbE}$ and $\mathrm{psbF}$ genes are separately transcribed from different regions of the plastid chromosome. Mol Genet, 1995, 246: 600604[DOI]

27 Pakrasi H B, Williams J G K, Arntzen C J. Targeted metagenesis of the $p s b E$ and $p s b F$ genes blocks photosynthetic electron transport: evidence for a function role of cytchrome $b_{559}$ in photosystem II . EMBO J, 1988, 7: 325-332 